\title{
La correspondance entre source et genre
}

\section{Réflexions sur les lettres de Gérard Machet, confesseur de Charles VII, évêque de Castres (vers 1380-1448)}

\section{Franck Collard}

Dans l'esprit des médiévistes, la personne de Nicole Pons est associée au monde des penseurs épris d'humanisme qui évoluaient entre l'Église et l'État sous Charles VI et Charles VII, et à leurs productions littéraires dont elle fut l'érudite spécialiste, mettant toute sa science à exhumer des textes souvent difficiles de lecture et de compréhension, et à les replacer dans une double perspective idéologique et culturelle. Le recueil épistolaire de Gérard Machet, objet de la présente communication, avait sans aucun doute attiré son attention, elle qui avait travaillé sur celui de Jean de Montreuil ${ }^{1}$, et qui connaissait fort bien un fameux correspondant de Machet, Nicolas de Clamanges ${ }^{2}$.

La préparation d'une biographie de Charles VII, dont Gérard Machet fut durant quasi trente ans le fidèle confesseur, ainsi qu'un intérêt pour l'épistolographie remontant aux recherches doctorales sur Robert Gaguin ${ }^{3}$, m'ont conduit à m'arrêter sur le recueil de lettres d'une importante figure de la première moitié $\mathrm{du} X \mathrm{X}^{\mathrm{e}}$ siècle français. Si le manuscrit est connu depuis longtemps - il n'avait évidemment pas échappé au marquis de Beaucourt dans sa monumentale Histoire de Charles VII ${ }^{4}$ - si, en 1968, Pierre Santoni lui a consacré sa thèse des chartes aujourd'hui libéralement mise en ligne dans une version révisée ${ }^{5}$, la

\footnotetext{
${ }^{1}$ Nicole Pons, «La présence de Coluccio Salutati dans le recueil épistolaire de Jean de Montreuil », Franco-Italica, 1, 1992, p. 9-24.

${ }^{2}$ Dario Cecchetti, «Nicolas de Clamanges e Gérard Machet: contributo allo studio dell'epistolario di Nicolas de Clamanges », Accademia delle scienze di Torino, Atti delle classe di scienze morali, 100, 1966, p. 133-191.

${ }^{3}$ Un article en a été tiré : Franck Collard, « La renaissance des lettres. La correspondance d'un humaniste français de la fin du XV $\mathrm{XV}^{\mathrm{e}}$ siècle, Robert Gaguin (1433-1501)», Bibliothèque d'Humanisme et Renaissance, 74, 2012, p. 19-33. Pour ne pas alourdir le propos, on se contentera de renvoyer le lecteur à la bibliographie de cet article sur l'histoire de l'épistolographie.

${ }^{4}$ Gaston du Fresne de Beaucourt, Histoire de Charles VII, Paris, Librairie de la Société bibliographique, 6 vol., 1881-1891; près de 20 renvois à l'index.

${ }^{5}$ Nous remercions très vivement l'auteur qui a accepté de mettre en ligne, à notre demande, les fichiers de ses transcriptions (https://halshs.archives-ouvertes.fr/halshs-01215771) après avoir déjà confié au site archives-ouvertes.fr son introduction (https://halshs.archives-ouvertes.fr/halshs-0060339).
} 
source n'a pas été étudiée dans les perspectives qui étaient celles de Nicole Pons.

C'est pourquoi la modeste contribution à l'hommage qui lui est rendu se propose d'examiner cette correspondance, de façon nécessairement succincte, en s'interrogeant non seulement sur les apports des lettres à la connaissance de l'époque et du milieu qu'elles mettent en scène, ainsi qu'à la connaissance des idées en circulation, mais aussi sur le statut documentaire d'un recueil dont il faut se demander s'il «fait œuvre ». Après un bref rappel sur l'auteur des lettres et sur le manuscrit les renfermant, le propos parcourra les grands domaines éclairés par les missives de l'évêque de Castres pour se clore sur une tentative de caractérisation du recueil: illustration de l'épistolographie humaniste française, intermédiaire entre le temps de Jean de Montreuil ${ }^{6}$ et celui de Robert Gaguin? Ou amalgame hétéroclite au statut ambigu et aux visées incertaines?

L'exigence de brièveté interdit de revenir en détail sur Machet et le manuscrit renfermant des lettres de sa confection. Ce qui suit résume en grande partie les données apportées par le travail très approfondi et méticuleux de P. Santoni dans l'introduction à son édition.

D'origine sans doute assez modeste (milieu de petits officiers seigneuriaux) et champenoise (Porcien), comme tant d'illustres penseurs dont le collège de Navarre permit de faire éclore les talents, Machet a bénéficié, à partir de 1391, des ressources matérielles et intellectuelles du «collège royal de Champagne », ainsi qu'il le nomme dans sa correspondance ${ }^{7}$. Né un peu avant 1380, il appartient à la génération des concampanistae ${ }^{8}$ qui suit celle de Gerson. Les études en théologie achevées en 1410 ont idéalement placé le gradué sur le marché des prébendes. La première est obtenue au chapitre de Notre-Dame de Reims. Elle est aussitôt échangée contre une prébende à Notre-Dame de Paris en 1410. Le théologien est aussi maître régent à

\footnotetext{
${ }^{6}$ L'Epistolario de Jean de Montreuil a été édité par Ezio Ornato, dans le premier volume de l'édition des opera de l'humaniste (Turin, G. Grappichelli, 1963). L'édition comprend 218 lettres, regroupées pour 202 dans le ms. lat. 13062 de la BnF.

${ }^{7}$ Lettre 23, Paris, BnF, ms. lat. 8577, fol. 10v (est donnée la localisation dans le manuscrit, faute d'une édition papier du travail de P. Santoni. Mais c'est à partir de sa transcription très sûre que les citations sont tirées et que la numérotation des missives est faite).

${ }^{8}$ Lettre 55, Paris, BnF, ms. lat. 8577, fol. 25 ; il désigne ainsi ses compatriotes champenois.
} 
l'université parisienne, si épris de son métier qu'en 1441, durant un assez long séjour à Paris, il le reprend temporairement ${ }^{9}$. Il est délégué à la collation des grades par le chancelier Gerson, lors du voyage de celui-ci à Constance en $1415^{10}$.

Les origines de sa position de confesseur du roi remontent à avant 1422 . Effrayé par les violences des Cabochiens qui avaient osé piller la demeure qu'il partageait avec Gerson, Machet avait pris nettement position contre les théories de Jean Petit en 1414 puis 1416. Il entra en contact avec le futur Charles VII qui avait mandé des chanoines de Notre-Dame à des fins d'aide financière. Le dauphin l'admit au conseil sans doute dès la fin de 1417 et utilisa ses services (son éloquence ?) pour négocier avec Jean sans Peur. Les événements de mai 1418 le chassèrent de la capitale et il se réfugia à Lyon, où il obtint un canonicat à la collégiale Saint-Paul. Durant deux ans, il vécut dans cette ville rejointe par Gerson un peu après lui. Recommandé au prince par le médecin de celui-ci, Jean Cadart ${ }^{11}$, il reçoit la charge de confesseur du régent à Mehun-sur-Yèvre, sans doute dans l'hiver 1420-1421. Il devait la conserver jusqu'à sa mort survenue en 1448, après des années d'épuisantes pérégrinations en des lieux jugés inhospitaliers ${ }^{12}$. Elle devait lui interdire de remplir personnellement la charge d'évêque de Castres reçue en 1432. Les fonctions exercées en permanence auprès du roi lui ont permis en revanche de connaître de près les événements du début du règne, qu'il livra sans doute au chroniqueur royal Jean Chartier lorsqu'il se rendit à Saint-Denis pour vérifier l'état d'avancement de sa chronique en $1441^{13}$. Il a siégé parfois au conseil, sans jouer toutefois un rôle de premier plan ni sortir des affaires ecclésiastiques et bénéficiales. Son office de confesseur lui valut d'être aussi administrateur du

\footnotetext{
${ }^{9}$ Lettre 101, ibid., fol. 29v.

${ }^{10}$ Sur tous ces éléments et ceux qui suivent, voir les premiers chapitres de l'introduction citée de P. Santoni.

${ }^{11}$ Lettre 354, Paris, BnF, ms. lat. 8577, fol. $92 \mathrm{v}$.

${ }^{12}$ Lettre 339, ibid., fol. 86v : in partibus Lothoringiae ubi aer intemperatus (dans les contrées lorraines à l'air rien moins que tempéré).

${ }^{13}$ Lettre 67, à Pierre de Versailles, évêque de Meaux, ibid., fol. 22 : [...] sermonem agebam cum rege super facto cronicarum examinandarum, qui voluit et ordinavit quod per vos et me una, quando vacare libebit, examinentur et visitantur; et hec notifficare curabo illi qui ad hec agenda deputatus est et electus («je conversais avec le roi au sujet des chroniques à examiner, ce qu'il a voulu et nous a ordonné de faire, à vous et à moi, quand nous en aurons le loisir ; et je vais m'occuper de faire savoir cela à celui qui a été désigné et choisi pour la tâche »).
} 
collège de Navarre et il a pris cette tâche à cœur ainsi que les affaires universitaires en général.

Ce personnage important du monde universitaire, ecclésiastique et curial a laissé un recueil de lettres conservé dans le manuscrit de la BnF coté latin 8577. C'est un manuscrit de format moyen, contenant aussi, à la fin, quelques autres écrits de Machet (dont un projet de discours pour défendre la Pragmatique Sanction) ou adressés à lui ${ }^{14}$. La correspondance couvre les folios 4-104 du codex puis le folio 106v. Le confesseur n'a pas tenu lui-même la plume, mais il est sans nul doute à l'origine du recueil. L'introduction à la table alphabétique des destinataires, contemporaine de la confection du recueil, dit bien le «livre» fait par lui ${ }^{15}$. On dénombre en tout 392 missives de toute taille, transcrites à partir de 1446 par un même copiste de la première à la $389^{\text {e }}{ }^{16}$. Les trois autres pourraient avoir été ajoutées une quinzaine d'années après la mort de Machet ${ }^{17}$.

Ce ne sont pas les lettres d'une vie. Hormis une douzaine de textes qui datent des années 1430, tout le reste a été rédigé entre 1441 et 1448 , soit à la fin de l'existence de l'épistolier, avec un énorme déséquilibre en faveur des années 1441-1442 (plus des deux-tiers des lettres) puis 1445 (86 lettres), alors que les années 1446-1448 n'en rassemblent que 45, un peu plus que les années 1443-1444. Point de lettres remontant au temps de la correspondance avec Nicolas de Clamanges ${ }^{18}$, on y reviendra, ni aux années 1420.

Confié à quelque secrétaire, le travail de copie a été effectué sans grand soin ni apprêt. Si l'écriture est assez lisible, quoiqu'irrégulière, les ratures ne sont pas rares ${ }^{19}$, non plus que les ajouts ou les corrections dans les marges. La numérotation est confuse et erronée faute d'une séparation claire entre les lettres successives. Commencée par une majuscule un peu plus haute, chaque lettre ne se distingue pas toujours de la suivante, au point d'être parfois

\footnotetext{
${ }^{14}$ Description précise du manuscrit dans le chapitre 12 de l'introduction de P. Santoni.

${ }^{15} \mathrm{BnF}$, ms. lat. 8577, fol. 2 : «S'ensuit la table ou repertoire de chascune lettre escripte en ce present livre fait par Gerart, evesque de Castres, confesseur du roy nostre seigneur. »

${ }^{16}$ Selon P. Santoni, introduction, chap. 12.

17 Ibid.

${ }^{18}$ P. Santoni, «Les lettres de Nicolas de Clamanges à Gérard Machet : un humaniste devant la crise du royaume et de l'Église ", Mélanges de l'École française de Rome. Moyen Âge-Temps modernes, 99, 1987, p. 793-823, ici p. 804.

${ }^{19} \mathrm{BnF}$, ms. lat. 8577 , fol. $5 \mathrm{v}$.
} 
fusionnée avec celle-ci (lettres $87-88^{20}$ ). À quelques exceptions (lettres 324, 326), le destinataire n'est mentionné qu'à la fin de chacune des missives. Il manque même dans une quinzaine de cas. La succession des lettres n'obéit pas à la chronologie. On observe un retour aux années 1432-1437 au folio 77, à partir de la lettre 315 jusqu'à la lettre 327. Le regroupement par lieu d'expédition n'est pas non plus évident, même si l'on peut repérer des noyaux homogènes. Pas non plus de regroupement par destinataires comme il peut s'en trouver chez Nicolas de Clamanges $^{21}$ : les renvois de la table montrent au contraire une grande dispersion des lettres adressées au même correspondant. On peut repérer tout au plus des regroupements thématiques. Le plus net est opéré autour des missives envoyées aux divers interlocuteurs diocésains de l'évêque de Castres, à son avènement en 1432 puis durant l'été $1441^{22}$.

Matériellement parlant, le recueil constitué par Machet et complété sans doute par un de ses proches, au début des années 1460, paraît assez en deçà de ce que l'on pourrait attendre dans ce milieu hautement lettré et curial. N'était-ce qu'un brouillon préparatoire à une belle édition, comme l'est le manuscrit parisien des lettres de Jean de Montreuil ${ }^{23}$ ? Impossible de le dire. C'est en tout cas un gisement documentaire dont il faut maintenant rapidement parcourir les domaines.

Telle qu'elle a été recueillie au milieu des années 1440, la correspondance du confesseur de Charles VII offre d'abord des vues sur la société ecclésiastique de l'époque, à commencer par l'auteur qui livre quelques données sur lui-même, ses origines, sa condition de confesseur royal et d'évêque absentéiste malgré lui, les rôles qu'il joue. La minutieuse édition de P. Santoni permet d'identifier presque tous les destinataires des lettres ainsi que les personnes auxquelles elles font allusion. La table du manuscrit, certes erronée (un destinataire peut apparaître sous deux noms ou ne pas apparaître du tout),

\footnotetext{
${ }^{20} \mathrm{Ibid}$., fol. 27v. Voir aussi fol. 71 : une main a écrit valete dans la marge à mi-hauteur du feuillet pour signifier la fin de la lettre numérotée 292 par le manuscrit ; mais celle qui commence ensuite, sans aucune marque de distinction, n'a pas été identifiée comme différente ; autre absence de délimitation aux fol. 76v, 91, si bien que la numérotation, peut-être effectuée par le copiste, présente un retard de cinq avec le nombre réel des lettres.

${ }^{21}$ P. Santoni, «Les lettres de Nicolas de Clamanges à Gérard Machet », art. cité, p. 804.

${ }^{22}$ Lettres 68-75 et 317-320, BnF, ms. lat. 8577, fol. 22 et suiv., 77 et suiv.

${ }^{23} \mathrm{BnF}$, ms. lat. 13062. Voir l'introduction d'Ezio Ornato à l'édition citée de l'« Epistolario » de Jean de Montreuil, p. XL-XLI.
} 
en mentionne 119. Se dessinent les réseaux relationnels d'un homme aux liens diversement étroits avec ses correspondants (près de la moitié n'est destinataire que d'une seule lettre) mais très forts avec ses compatriotes champenois (la première lettre est dédiée à un chanoine de Notre-Dame de Reims), ses condisciples navarristes, les clercs lyonnais qui l'ont accueillis en 1418, divers chapitres (en premier lieu, évidemment, celui de Castres) et divers chanoines (25 destinataires), des évêques ou archevêques (28 destinataires), des abbés ou des prieurs, essentiellement du royaume, hormis le souverain pontife ${ }^{24}$ et ses légats. Cet ensemble représente plus des trois quarts des correspondants. Sept d'entre eux ont reçu chacun dix lettres au moins. Le mieux pourvu est Jacques de Cerizy, navarriste, secrétaire de Gerson, chanoine de Saint-Paul de Lyon, avec 34 missives reçues. Suit, avec moitié moins, un laïc, Adam de Cambrai, premier président du parlement de Paris, ex-navarriste. Mais les non-clercs ne constituent qu'une faible proportion $(10 \%)$ des gens à qui Machet écrit. Ses interlocuteurs sont avant tout des chanoines prébendés devenus parfois évêques et les officiers ecclésiastiques de son diocèse. Le recueil montre bien, dans l'Église, les jeux d'influence, de recommandation, de solidarités et de faveurs au cœur desquels est celui qui dit avoir quotidiennement, et pour cause, l'oreille du roi ${ }^{25}$.

Le second champ d'investigation explorable à partir de la correspondance de Machet est celui de l'histoire de l'Église. Il est question des affaires ecclésiastiques dans pratiquement 200 lettres. Elles les abordent à plusieurs échelles. Celle de l'Église universelle d'abord, avec le conflit entre pape (Machet aime peu Eugène IV, davantage le théologien Nicolas $\mathrm{V}^{26}$ ) et concile :

\footnotetext{
${ }^{24}$ Lettres 63, 112, 315, 362, 367 et 382, BnF, ms. lat. 8577, fol. 21, 31v, 77, 94v, 95v, 103v.

${ }^{25}$ Lettre 35 à l'évêque de Noyon, ibid., fol. 14 : merito letus et gaudens, confestim regiam majestatem adii, et morigerare cupiens piis votis vestris et desideriis reverende paternitatis vestre, postulans ut omnia beneficia jamfati servitoris in regalia conferret, quod facile annuit rex, memor vestre reverende paternitatis («me réjouissant à bon droit et plein de liesse, j'allai aussitôt auprès de la majesté royale, désireux de complaire à vos pieux vœux et désirs, révérend père, et demandant la collation en régale de tous les bénéfices dudit serviteur, ce à quoi le roi a consenti sans faire de difficulté, se souvenant de votre paternité à révérer »). Voir aussi lettre 103, fol. 30 : Non omisi, suscepta cartula vestra, festinanter regem adire pro illo officio, quod obtinui si vacat (« Je ne me suis pas fait faute, votre brève lettre reçue, de me hâter auprès du roi pour vous obtenir cet office en cas de vacance »).

${ }^{26}$ Lettre 367, ibid., fol. 96 : in cujus [papae] assumptione nova lux oriri visa est, gaudium et exultatio cunctis theologie professoribus ( « l'élévation de ce pape a semblé faire naître une nouvelle lumière, motif de joie et d'exultation pour l'ensemble des professeurs de théologie »).
} 
hostile aux dérives de Bâle ${ }^{27}$, mais convaincu de la légitimité représentative du concile $^{28}$, il veut un troisième concile général, à tenir en France, occasion pour Charles VII de rendre au roi de France la direction de la chrétienté ${ }^{29}$. Une trentaine de lettres parlent de l'indispensable reformatio generalis. Machet se soucie aussi de l'Église gallicane dans ses rapports avec Rome la vénale (venalia sunt omnia apud illos ${ }^{30}$ ) et avec le roi (une quinzaine de lettres), sujet qui enflamme le confesseur particulièrement indigné que le nonce apostolique ait soutenu que la Pragmatique, à laquelle il est viscéralement attaché ${ }^{31}$, sentait l'hérésie ${ }^{32}$. Enfin, le pasteur absentéiste mais consciencieux qu'est le prélat de Castres $^{33}$ aspire ardemment à la réforme de l'église diocésaine qui le retient dans plus d'une cinquantaine de lettres où il se montre en particulier très vigilant avec ses différents vicaires généraux au comportement rien moins qu'exemplaire ${ }^{34}$.

${ }^{27}$ Lettre 42, ibid., fol. 15v: Diu perdurare non potest congregatio illa Basiliensis, suo mucrone confodietur et dissipabitur in non modicam lesionem honoris debiti sacratissimis sinodis, que maculam in gloria sua posuit, ita ut irrideant sabbata ejus plurimi. ( Cette assemblée de Bâle ne peut perdurer plus longtemps, elle tombera sous ses propres coups et sera dispersée après avoir gravement porté atteinte à l'honneur dû aux saints synodes, et entâché tant sa gloire que maintes gens moquent ses honteuses réunions »).

${ }^{28}$ Lettre 160, ibid., fol. $41 \mathrm{v}$ : il se réfère à l'auctoritati conciliorum generalium que Ecclesiam [...] representare nulli sane sapienti dubium est («l'autorité des conciles généraux dont aucun sage ne doute sérieusement qu'ils repésentent l'Église »).

${ }^{29}$ Lettre 168, ibid., fol. 44 : Rex satis afficitur observare Pragmaticam Sanctionem [...] Convocationem tertii concilii laudat, probat et necessariam ita arbitratur (« Le roi est suffisamment disposé à observer la Pragmatique Sanction [...]. Il loue, approuve et juge nécessaire la convocation d'un troisième concile »).

${ }^{30}$ Lettre 46, ibid., fol. 17 : « tout est à vendre chez ces gens-là ».

${ }^{31}$ Il est sans doute l'inventeur de la fausse Pragmatique de saint Louis, à quoi son projet de discours de 1445 fait allusion.

${ }^{32}$ Lettre 217 et 278, Paris, BnF, ms. lat. 8577, fol. $53 \mathrm{v}$ et $67 \mathrm{v}$, à l'évêque de Montauban dont Machet veut obtenir qu'il dépose sur l'injure du légat pro honore regie celsitudinis, regni totius et Ecclesie gallicane («afin de laver l'honneur du roi, du royaume et de l'Église gallicane »).

${ }^{33}$ Lettre 62, ibid., fol. 20v : precipue a die qua assumptus fui immeritus ad dignitatem episcopalem ecclesie Castrensis, desideravi ordinem dare, mores in melius refformare religiosorum meorum ( «avant tout, du jour où je fus élevé sans le mériter à la dignité épiscopale, à la tête de l'Église de Castres, j’ai voulu remettre de l'ordre et réformer les mœurs de mes clercs »).

${ }^{34}$ Lettre 243, au prieur-mage du chapitre, ibid., fol. 59v, à propos de Guillaume Boucher : Nundum illum vidi aut audivi, quia alibi declinavit pro negotiis propriis et familiaribus. Sua etenim voluntate, non mea instantia recessit a vobis [...] («je ne l'ai encore ni vu ni entendu du fait qu'il s'est rendu ailleurs pour ses propres affaires et ses familiers. C'est de sa propre volonté et non sur mes instances qu'il s'en est retourné »). 
Près de 70 lettres au bas mot sont ainsi, en partie ou en totalité, consacrées à des affaires bénéficiales, administratives ou judiciaires. Mais la correspondance de Machet est intéressante aussi du point de vue de l'histoire religieuse. Machet voue une grande affection aux Célestins (pas moins de sept lettres) et aux Chartreux ${ }^{35}$. Une bonne trentaine de lettres, et parmi les plus développées, présentent des considérations d'ordre spirituel. L'influence de Gerson n'est pas loin quand, hostile aux abus de l'excommunication ${ }^{36}$, il écrit qu'absoudre et sauver valent mieux que condamner ${ }^{37}$.

Connexes à celles de l'Église, les affaires de l'Université retiennent aussi largement l'attention de l'ancien maître régent en théologie. Une grosse soixantaine de lettres traitent des affaires parisiennes ${ }^{38}$, et tout particulièrement navarristes. La gouvernance du collège royal préoccupe son administrateur, de même que la nécessité de former des théologiens pour combattre l'hérésie ${ }^{39}$. La brigue nuit à la qualité des cours ${ }^{40}$. Là aussi la réforme est le maître mot, ce

${ }^{35}$ Exemples, lettres 24 ou 68, ibid., fol. 10v et 22. La réputation des frères de l'ordre de saint Bruno est très préservée en ce temps. Pour ce qui est des Célestins, faut-il voir un attachement de nature plus politique à un ordre très lié à la royauté française qui l'a pour ainsi dire créé contre Rome, voire à la maison d'Orléans dont le chef assassiné en 1407 fréquentait beaucoup le couvent parisien de l'ordre ?

${ }^{36}$ Lettre 97, ibid., fol. 29: Odio et abhominatus sum tales excommunicationes nec volo ditari de sanguine ovium mearum («je hais et exècre de telles excommunications et me refuse à m'abreuver du sang de mes brebis »).

${ }^{37}$ Lettre 314, à l'official de Castres, ibid., fol. 76v : Ante omnia, frater, precor, obsecro in mansuetudine et caritate, non ficta opera perficere, saluti animarum providere; tarde, tardius, tardissime ferre sententiam excommunicationis, nisi evidens contumacia precesserit. Prona inveniatur caritas vestra magis absolvere et salvare quam condempnare («Avant toute chose, frère, je te prie et t'adjure de pourvoir au salut des âmes dans la mansuétude et la charité, non d'accomplir des œuvres feintes ; de ne prononcer de sentence d'excommunication qu'en en retardant au maximum le moment et seulement en cas de contumace évidente. Que votre charité soit trouvée plus encline à absoudre et sauver qu'à condamner »).

${ }^{38}$ Lettre 125, ibid., fol. 34, sur les conditions d'attribution des bourses.

${ }^{39}$ Lettre 140, ibid., fol. 38-38v.

${ }^{40}$ Lettre 214, ibid., fol. 53 : [...] subaudio nonnullos decertantes pro rectoriis universitatis aut officio procuratoris; que quamtum detrimenti studiosis viris afferant ipsi nostis. Vidi et in diebus meis, et hec agnoscens statui animo meo, dum exercebar in ginasio theologico, numquam ambire ad hec officia [...] ( «j'ai eu vent que quelques-uns se battaient dans l'Université pour être recteurs ou procureurs, chose ô combien au détriment des hommes d'étude, comme vous le savez. Je l'ai vu et, de mon temps, lorsque j'exerçais à la faculté de théologie, le sachant, j'ai décidé de ne jamais ambitionner d'occuper ces fonctions »). 
qui n'empêche pas l'ancien élève du collège de Navarre d'exalter son florissement singulier au milieu de la désolation des écoles parisiennes ${ }^{41}$.

Autre domaine attendu d'exploitation du document, le champ politique. Confesseur du roi jamais tombé en disgrâce, contrairement à bien des gens de l'entourage de Charles VII ${ }^{42}$, Machet donne dans sa correspondance quelque écho aux affaires du Siècle, sans toutefois beaucoup sortir de propos convenus et réitérés sur le malheur des temps ${ }^{43}$. Une dizaine de lettres évoquent la situation internationale (germanique en particulier), un peu plus de vingt-cinq font référence à des évenements français ou dauphinois - puisque Machet compte parmi ses correspondants le précepteur du dauphin, Jean Majoris.

Quoiqu'il siège parfois au conseil, le confesseur du monarque ne livre guère d'analyse poussée de la situation politique. Il effleure la rébellion du fils qu'il n'aime guère ${ }^{44}$ - contre le père en $1440^{45}$. Assez avare en notations précises sur la personne royale ${ }^{46}$, il use sur le souverain de propos conventionnellement laudateurs : rex christianissimus et columpna firmior

${ }^{41}$ Lettre 101, ibid., fol. 30 : Collegium regale solum ibi floret; cetera jacent inculta, evacuata studentibus [...] («Seul le collège royal est ici florissant; les autres sont à l'abandon, vidés de leurs étudiants »).

${ }^{42}$ Pierre-Roger Gaussin, «Les conseillers de Charles VII (1418-1461). Essai de politologie historique », Francia, 10, 1982, p. 67-130: seulement neuf trimestres de présence cumulée pour Machet entre 1421 et 1448, ce qui place le confesseur très loin derrière l'évêque de Sées puis Maguelonne, Robert de Rouvres (73 semestres cumulés).

${ }^{43}$ Lettre 133, BnF, ms. lat. 8577, fol. 35v : O mores ! O tempora! 0 dies mali !

${ }^{44}$ Lettre 239, ibid., fol. 58v, sur l'impulsivité inconsidérée de Louis, à qui elle a valu une blessure.

${ }^{45}$ Lettre 30, lettre à Adam de Cambrai, ibid., fol. 12v, très allusive au demeurant, citant Matthieu, XXIV, 22: Sane comperimus diebus nostris malis illud evvangelicum et verbum Veritatis, Christum loquor: «Nisi breviati essent dies, non esset salva omnis caro»; et rursum : "Exurgent filii in parentes » («Nous avons assez vérifié par les mauvais temps qui courent cette parole évangélique et de vérité du Christ : "Si ces mauvais jours n'avaient pas été diminués, nul n'aurait survécu”; et aussi : "Les fils se lèveront contre leurs pères" »).

${ }^{46}$ Lettre 231, ibid., fol. 56v, sur les forces un peu déclinantes du roi : Certum, vires non suppetunt ut olim, qui utpote (et melius nosti) tenuis est et minus rigide probatur temperature, quam complecionem ita vocitatis ( «Sans conteste, il n'a plus les forces d'autrefois et c'est naturel de la part d'un homme qui (et tu le sais mieux que moi) est chétif et de moins solide de tempérament, ce que vous appelez la complexion ») ; lettre 229 , ibid., fol. 56 , sur sa colère puis son apaisement à l'égard de son médecin Beschebien : sermo habitus est de persona vestra, me solo cum rege manente, et inveni eum post habitum colloquium animo placatum et longe sequestratum a passione illa que tam diu eum detinuit («Nous avons parlé de vous, seul à seul avec le roi, et je l'ai trouvé apaisé après la conversation, et grandement libéré de cette passion qui l'a si longtemps possédé »). 
corporis ecclesiasticii ${ }^{47}$, c'est un homme qui, écrit-il en 1448, croît chaque jour en sagesse ${ }^{48}$. Il défend fermement l'Église gallicane, conforté en cela par son confesseur $^{49}$. Faisant refleurir sur son passage le royaume dévasté ${ }^{50}$, il met toute son énergie à chasser l'ennemi ${ }^{51}$, quitte à ne pas réunir le conseil durant de longues périodes ${ }^{52}$. Mais la charge qu'il porte est si écrasante que cela apitoie Machet ${ }^{53}$. On sait bien que ses lettres ne pouvaient être dépositaires des secrets de la confession. Par conséquent, en partie à cause du devoir de réserve que lui impose son office ${ }^{54}$, les apports du recueil sont singulièrement limités

\footnotetext{
${ }^{47}$ Lettre 340, ibid., fol. 87 : « roi très chrétien, colonne la plus solide du corps de l’Église ».

${ }^{48}$ Lettre 380, ibid., fol. 100 : Rex noster etate et sapientia proficit, negotiis propriis vigilanter attendit (« Notre roi progresse en âge et en sagesse, attentif et vigilant à ses propres affaires »).
}

${ }^{49}$ Lettre 128, ibid., fol. 34v : Ista ingessi auribus regis, qui suam Pragmaticam Sanctionem in terminis suis deffendere et protegere deliberatus est, ut ait. [...] Sicut regi narrabam, providendum est saluti animarum regni sui, que pereunt ob infectionem pestis jamdicte symonie, que pecunias exhaurit sine misericordia totius istius dominationis ( «J'ai rapporté ces choses aux oreilles du roi qui est bien décidé à défendre et protéger la Pragmatique Sanction dans son intégralité, comme il le dit. [...] Comme je le disais au roi, il faut pourvoir au salut des âmes de son royaume, mises en péril par la peste simoniaque qui puise des deniers sans miséricorde aucune »). Machet estime que la provision aux bénéfices par Rome résulte de la simonie car rien ne s'obtient sans argent à la Curie.

${ }^{50}$ Lettre 20, ibid., fol. 10: Adveniente autem regia providentia, nunc pace optatissima regiones perfruuntur, terras incolunt agricole, propulsatis armatis predonibus («Par la providence royale et la paix tant désirée, les contrées du royaume fleurissent, les agriculteurs cultivent les terres, maintenant que les brigands de l'armée ont été chassés »). La lettre datée d'avril 1441 par son éditeur est bien antérieure aux réformes militaires de 1445, mais elle prend peut-être en compte celle de 1439.

${ }^{51}$ Lettre 56, ibid., fol. 19v : Cor regis super ceteros firmatum est et stabile, ita ut in admiratione omnium habeatur. Imperturbatus visus est transeuntibus Anglicis in Insulam Francie, quasi solus imperterritus et animo audens. Non sic ceteri, non sic qui fugiebant a facie arcus [...] (« Le cœur du roi est, plus que d'autres, ferme et stable, si bien qu'il fait l'admiration générale. Impavide devant les Anglais présents en Île de France, quasi seul à n'en pas être perturbé, l'audace à l'esprit, pas comme ceux qui fuyaient devant les arcs »).

${ }^{52}$ Lettre 163, écrite au moment de la campagne de Tartas, ibid., fol. 42v : Rex hic stat, absente consilio a tribus mensibus (« Le roi reste là, sans avoir tenu un conseil depuis trois mois »).

${ }^{53}$ Lettre 54, écrite au moment du siège de Pontoise, ibid., fol. 18v-19 : Nostis onera importabilia et vix credibilia que opportet regem sufferre. Video oculis et vix a lacrimis contineo. Jugis est conflictus ejus, jugis pugna et hoc solum pertimescit, ne desint sumptus ad perficienda ea que tam audenter et tam necessarie inchoavit, ita ut admirentur omnes animi constantiam, indefessos labores anime et corporis («Vous savez les charges lourdes et à peine croyables qu'il faut supporter au roi. Je le vois de mes yeux, peinant à retenir mes larmes. Sa querelle est sans fin, son combat aussi et il ne craint qu'une chose, c'est que viennent à manquer les fonds pour mener à terme ce qu'il a commencé si audacieusement et si nécessairement, à l'admiration de chacun devant sa constance dans la peine de corps et d'esprit qu'il se donne inlassablement »).

${ }^{54}$ Sur certains points comme les trêves de Tours ou l'éventuelle abrogation de la Pragmatique Sanction, Machet dit mieux aimer se taire (lettres 228, 236, ibid., fol. 55v et 58). 
sur les amours royales ${ }^{55}$, la cour, les rivalités, les luttes d'influence, à quelques mentions de disgrâce près, comme celle de l'évêque de Laon Guillaume de Champeaux ${ }^{56}$, ou celle du médecin Pierre Beschebien pour incapacité à soulager le roi ${ }^{57}$. Contrairement à un Alain Chartier, Machet ne donne cependant pas dans l'anti-curialisme véhément. Les courtisans ne l'intéressent pas, sinon pour dire qu'ils jalousent son ami l'argentier Jacques Cœur, car la cour est par définition en quête de mutations permanentes ${ }^{58}$.

La correspondance de Machet est enfin d'un apport certain pour l'histoire culturelle et intellectuelle de ce temps, situé entre le premier et le deuxième humanisme français. Même si les lettres à sujet purement intellectuel ne prédominent pas dans le recueil (une petite trentaine), celui-ci reflète les échanges de lettrés épris de poésie et de rhétorique. Le recueil présente un intérêt particulier par les réflexions multiples, certes assez topiques, de Machet sur l'amitié, son entretien, ses manifestations ${ }^{59}$. Il illustre aussi la place de la pensée de Gerson dans la culture française du temps. Machet évoque le recueil de ses œuvres ${ }^{60}$, la copie d'un de ses textes, le Testamentum peregrini ${ }^{61}$. En revanche, les lettres du confesseur ne donnent aucun écho à la compétition culturelle avec l'Italie, sujet qui avait tant préoccupé les premiers humanistes français comme l'ont montré les travaux pionniers de Fr. Simone et de ses

\footnotetext{
55 Peut-être une allusion aux turpitudes royales avec Agnès Sorel dans la lettre 263 au prieur des Célestins de Paris, fol. 63v : Commandari precor sancte vestre congregationi, que et dominum nostrum regem commandatum teneat die ac nocte, ut non involvat cum procella hujus naufragii, nequam seculum loquor ( « Je prie d'être dans la recommandation de votre sainte congrégation et que celle-ci tienne le roi notre sire nuit et jour en sa recommandation de sorte qu'il ne s'abîme pas dans le naufrage de cette tornade, je veux dire cette débauche »).

${ }^{56}$ Lettre 61, ibid., fol. 20v.

${ }^{57}$ Lettre 229, ibid., fol. 55v-56 et deux autres lettres $(253,298)$.

${ }^{58}$ Lettre 266, ibid., fol. 64 : more suo prudenter agere novit, non sine multorum invidia, ut solet mundus et curia mendax, que nova semper expetit omnia mutari, quod rarissime in melius contingere vidi [...] (« À sa façon, il a su agir prudemment, non sans exciter l'envie de beaucoup, comme d'habitude dans les affaires du monde et de la cour, où règnent la tromperie et la sempiternelle attente de nouveautés et de mutations générales, très rarement synonymes d'amélioration selon mon expérience »).

${ }^{59}$ Exemples, lettre 197 ou 354 ou 372, ibid., fol. 49v ou 92 ou 97v.

${ }^{60}$ Lettre 330 et 331 , ibid., fol. $83 \mathrm{v}-84$.

${ }^{61}$ Lettre 294, ibid., fol. 71.
} 
émules ${ }^{62}$. Une lettre parle bien de Pétrarque, mais sans aucune perspective polémique et en écorchant le titre d'une de ses œuvres dont il ne peut plus s'arracher : le Remedium fortuitorum (sic) que Machet a relu et où il a trouvé de la consolation ${ }^{63}$. Peut-être dans les années 1440 la querelle était-elle retombée. Mais c'est aussi un élément à prendre en compte pour dégager la signification de la confection du recueil, ou du moins en cerner les enjeux.

Quelle finalité, quelle ambition le recueil des lettres que Gérard Machet conçut à la fin de sa vie poursuivait-il ? Familier des premiers humanistes français, l'évêque de Castres ne pouvait ignorer la reviviscence de l'épistolographie illustrée par les épîtres familières de Pétrarque, si présentes à l'esprit de son ami Clamanges, ni la démarche épistolographique très élaborée de ce dernier ${ }^{64}$. Se place-t-il dans leur sillage ? Son «epistolario»a-t-il une prétention littéraire?

Se pose d'abord la question de la constitution même du recueil, aux deux sens de son entreprise et de sa consistance. La première est tardive, voire fortuite (occuper le long séjour de la cour à Châlons ?), elle s'avère assez peu pensée, quand on observe la localisation des missives dans le manuscrit, fruit d'un laborieux agrégat bien expliqué par P. Santoni ${ }^{65}$. Et elle n'est rien moins que systématique. Des lettres des années 1441-1448 dont l'existence est attestée par des passages de plusieurs missives du recueil ${ }^{66}$, sont absentes de celui-ci. Une certaine sélectivité semble donc avoir présidé à sa démarche qu'il est par conséquent impossible de réduire à la confection d'un simple registre de

${ }^{62}$ Franco Simone, «Influenze italiane nella formazione dei primi schemi della storiografia letteraria francese », Lettere italiane, 17, 1965, p. 275-29; Dario Cecchetti, Il primo umanesimo francese, Turin, 1987.

${ }^{63}$ Lettre 84 à Guillaume Saignet, BnF, ms. lat. 8577, fol. 27 : Lego, suavissime domine et vere amabilis, Franciscum Petrarche, laureatum poetam, vestre, scio, prudentie familiarissimum. Avidius autem relegi "De remediis fortuitorum", qui utique adeo insedit animo ut vix avelli possim, multam in eo consolationem recipiens ( «Je lis, maître plein de douceur et d'amabilité, le poète lauréat François Pétrarque dont je sais familière votre prudence. J'ai relu avec plus d'avidité les Remèdes de Fortune qui m'a occupé tellement l'esprit que je pourrais difficilement m'en séparer, si grande est la consolation que je trouve à sa lecture »).

${ }^{64}$ D. Cecchetti, «Nicolas de Clamanges e Gérard Machet », art. cité (n. 2), estime que Machet est celui qui a réuni les lettres de Clamanges contenues dans le ms. Ottoboni latin 260 de la BAV.

${ }^{65} \mathrm{P}$. Santoni, introduction, chap. 12, à partir d'un minutieux examen des cahiers, c'est durant le séjour du confesseur à Châlons, en 1445-6, que le principe du recueil aurait été conçu, et son noyau originel constitué des lettres écrites alors. Il aurait été ensuite enrichi par des lettres antérieures et postérieures.

${ }^{66}$ Lettres 21, 97, 101, BnF, ms. lat. 8577, fol. 10, 29 et 30. 
correspondance. Machet se montre soucieux de rassembler des missives égarées qu'il juge dignes de figurer dans son « epistolario », comme cette lettre sur l'amour dont il réclame par deux fois la copie à Jacques de Cerizy ${ }^{67}$.

Mais le critère qualitatif du contenu des lettres comme facteur de leur sélection trouve vite ses limites. D'une part, à moins qu'elle ne résulte d'une perte consécutive à la fuite de Machet de Paris en 1418 et de sa décision tardive de rassembler ses lettres, l'absence de toute missive adressée à Nicolas de Clamanges (mort en 1437) ne manque pas d'intriguer, alors que le recueil de celui que Clamanges tenait en haute estime ${ }^{68}$ eût été ennobli de leur insertion $^{69}$. L'épistolier n'a pas cherché à se rattacher à la haute figure de l'épistolographie que fut Nicolas. D'autre part, la consistance du recueil est des plus hétéroclites, ce qui, certes, peut se retrouver dans l'« epistolario » de Jean de Montreuil ou la correspondance de Nicolas de Clamanges, mais atteint un degré assez frappant dans le manuscrit latin 8577 de la BnF. En effet, le lecteur y trouve aussi une dizaine de billets prosaïques ou triviaux de quelques lignes et dépourvus de toute valeur autre qu'informative, l'un portant par exemple sur la transmission du double d'une quittance ${ }^{70}$.

${ }^{67}$ Lettres 189 et 346, ibid., fol. 48 : Dudum pagellam direxi vestre caritati super amoris violentia, quam inter mea scripta non invenio. Copiam reddite, ut reservetur apud me (« Il y a quelque temps, j'ai adressé à votre charitable personne une lettre sur la violence de l'amour que je ne retrouve plus parmi mes écrits. Renvoyez-m'en une copie que je mette de côté pour moi »); fol. 90 : Memini, frater, olim scripsisse vestre devotioni ut si penes se reservatam inveniret litteram a me olim descriptam super amore, quomodo ligat, vulnerat, languere facit et deficere, ut coppiam remittat dum vacaverit, quia inter cetera opera mea minime reperio ( «Vous vous souvenez, frère, que j'ai autrefois écrit à votre dévote personne que si elle retrouvait dans ses affaires la lettre sur l'amour écrite autrefois de ma main, et disant comment il lie, blesse, fait languir et défaillir, elle en remette une copie pour autant qu'elle en ait le temps, car je ne la retrouve pas parmi mes autres œuvres »).

${ }^{68}$ Voir D. Cecchetti, «Nicolas de Clamanges e Gérard Machet », art. cité (n. 2), p. 183, première lettre du recueil du Vatican adressée Viro magne litterature et circumspectionis, Magistro Girardo Macheti, canonico parisiensi, domino et magistro suo carissimo ( « À maître Gérard Machet, homme très lettré et prudent, chanoine de Paris, son très aimé seigneur et maître »).

${ }^{69}$ P. Santoni, «Les lettres de Nicolas de Clamanges à Gérard Machet », art. cité (n. 18). L'auteur montre que Machet envoya plusieurs lettres à Nicolas de Clamanges avec qui il eut des relations heurtées, à cause des positions très tranchées du second. Mais il paraît assez improbable que leurs différends aient détourné Machet d'insérer ses missives à Clamanges. Ce dernier aurait pu renvoyer copie de ses lettres au confesseur du roi.

${ }^{70}$ Lettres 18 , BnF, ms. lat. 8577, fol. 9v. Le destinataire est dûment inscrit dans la table du début du manuscrit. 
Pourtant, Machet fait montre d'une réelle sensibilité à la force rhétorique des lettres, consacrant fréquemment son propos à des considérations sur les joies immenses qu'en procurent lecture et relecture, au plaisir intense que suscite l'officium litteratorium ${ }^{71}$, aux vertus de l'échange épistolaire qui présentifie littéralement le correspondant. Ea enim vis est scriptorum ut absentes sua virtute presentes exhibeant, s'extasie-t-il ${ }^{72}$. Mais il constate aussi, dans plusieurs lettres de l'année 1447, l'incapacité des mots à exprimer les sentiments les plus profonds, que la seule présence physique permet d'épancher ${ }^{73}$. Le confesseur du roi développe ce que l'on pourrait appeler une philosophie de la lettre qui rejoint, sans surprise, celle des Anciens et des épistoliers humanistes.

Il est moins patent qu'il en développe une esthétique. Rien n'indique qu'il ait suivi, en rassemblant ses lettres, une démarche littéraire, ni qu'il ait voulu mettre cette démarche au service d'un genre rénové par l'humanisme. Son recueil est dépourvu de tout prohème. Dans la lettre où il parle de Pétrarque ${ }^{74}$, il n'évoque pas sa correspondance, pas plus qu'il ne se réfère à des modèles antiques ou plus récents. Machet ne développe aucune théorie formelle de la lettre. Nulle part il ne présente les canons de l'épistolographie. Certes, il estime qu'une lettre ne doit pas excéder une certaine taille ${ }^{75}$, mais il fait varier grandement leur longueur, proche en cela de Jean de Montreuil. Mesurée d'après l'édition Santoni, elle va de moins de cinq lignes (25 lettres) à plus de cent (2 lettres), avec une majorité (278) de lettres ne dépassant pas les 20 lignes, hors adresse.

\footnotetext{
${ }^{71}$ Lettre 161, ibid., fol. 42 : placet hoc opus, juvat hoc officium litteratorium peragere (« la chose me plait, j'ai plaisir à remplir cet office littéraire »).

${ }^{72}$ Lettre 312, ibid., fol. 76. C'est la force de l'écrit que de pouvoir rendre les absents présents. De nombreuses autres lettres exposent cette idée ancrée dans toute l'histoire de l'épistolographie.

${ }^{73}$ Exemple lettre 354, ibid., fol. 92 : Grata sunt, numquam negabo, munera litterarum, federa ista carioris amicitie, verum nichil gratius, nil desiderabilius amici presentia, que sola satiare potest fervens desiderium, implere cor meum petens et querens labiorum osculum, vivas audire et reddere voces,

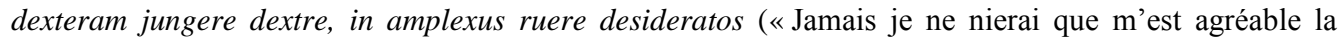
rédaction des lettres, ces liens entretenant la plus chère amitié, mais rien n'est plus agréable, plus désirable que la présence de l'ami qui seule peut satisfaire un brûlant désir et emplir mon cœur aspirant au baiser des lèvres, à entendre les voix, y répondre, à donner la main, à s'embrasser »).

${ }^{74}$ Lettre 84, ibid., fol. 27.

${ }^{75}$ Lettre 321, ibid., fol. 80v : Excessi forsan epistule modum («J'ai peut-être dépassé la taille d'une lettre $\gg$ ).
} 
Le confesseur du roi énonce quelques considérations linguistiques. Dans une lettre à Adam de Cambrai, il juge peu souhaitable l'emploi mêlé du latin et de la langue vulgaire ${ }^{76}$. Cette défense et illustration de la latinité se rencontrent à plus d'un endroit ${ }^{77}$. Mais elle n'est pas inconditionnelle - la lettre destinée aux «seurs mineuretes», à savoir les clarisses de Castres, est en langue vernaculaire $^{78}$. Et elle est curieusement contredite par le fait que le recueil s'ouvre par une longue lettre en français sur la musique sacrée. Surtout, la langue de Machet ne reflète en rien l'idéal d'un latin « restitué » et classicisant. Elle est, comme il le dit à Adam de Cambrai, celle des clercs et non celle des militaires, classification rien moins que littéraire ${ }^{79}$. Si elle peut viser des effets rhétoriques (exclamations de désolation ou d'indignation, exhortations véhémentes), la correspondance du confessseur ne présente pas de recherche lexicale, stylistique ou syntaxique telle que l'épistolographie néo-antique les prônait. Point d'interrogation sur l'emploi du tutoiement ou du voussoiement, l'épistolier use du second sans commentaire. Aucune interpretatio romana des termes administratifs ou des toponymes. La manière même dont il désigne ses missives est très significative : très souvent pagella, parfois scriptura, littera ou cartula, rarement epistola, terme pourtant consacré par la tradition biblique comme par la tradition classique, mais employé seulement par Machet pour dénommer l'épître reçue de l'ex-médecin de Charles VII, Jean Cadart ${ }^{80}$.

\footnotetext{
${ }^{76}$ Lettre 146, ibid., fol. 39v: Qualem dixerim aut vocitaverim pagellam vestram novissimam prorsus ignoro. Nampe, si a fine nomen accipiat, latinitate clauditur; si a parte majori denominatio fiat, sermo vulgaris est. Si autem rescriberem aut latinum totum aut vulgare, imparem vicem merito qui viderint me reddere dicent («Comment appeler ou qualifier votre dernière lettre, je ne sais vraiment dire. Car à s'en tenir à sa fin, elle ressortit à la latinité ; mais si l'on se fie à la majeure partie du contenu, il s'agit d'un discours en vulgaire. Si j'y répondais, ce serait soit totalement en latin, soit en vulgaire, ceux qui l'auront vue diront à juste titre que je me suis rendu inégal »).

${ }^{77}$ Lettre 80, ibid., fol. 25v : Duas post dies paucos a vobis accepi litteras : unam materna seu gallica lingua conscriptam ; alia latina erat, quam potius laudo et probo [...] («J'ai reçu en eu de jours deux lettres de vous, l'une écrite en français, la langue maternelle, l'autre en latin, plus louable et digne d'approbation »).

${ }^{78}$ Lettre 75, ibid., fol. 24v. Observons que ces sœurs étaient sans doute de langue d'oc et que l'emploi de la langue d'oïl par Machet ne garantit pas l'intelligibilité de sa lettre.

79 Lettre 146, ibid., fol. 39v: Si partita fuerit, non est clericalis habitus sed varia et peregrina conscriptio, et forsan potius militaris quam clericalis dici debuerat ( «Si elle a été écrite en deux langues, ce n'est pas par habitude de clerc, mais à cause d'une rédaction disparate et empruntée, relevant peut-être plus du style militaire qu'ecclésiastique »).

${ }^{80}$ Lettre 231, ibid., fol. 56v : disertissima et sane elegantissima epistola (« lettre très diserte et élégante »).
} 
Certes, comme pour les recueils des lettres de Nicolas de Clamanges ${ }^{81}$, les adresses, portées à la fin, sans doute de la malencontreuse initiative du scribe, sont dans $85 \%$ des cas formulées avec un soin particulier, dotées notamment d'épithètes superlatives de courtoisie au datif, conformément aux canons pétrarquiens ${ }^{82}$, épithètes parfois étendues sur deux ou trois lignes pour mettre en valeur l'érudition, l'éloquence ou le raffinement de leur destinataire ${ }^{83}$. Selon les préceptes humanistes, les éléments les plus prosaïques des lettres ont été sinon systématiquement, du moins très fréquemment enlevés, par exemple les dates et les lieux de rédaction, qui manquent dans l'écrasante majorité des missives recopiées (respectivement 382 et 303 sur 392). Mais quand la date est mentionnée, aucune recherche de formulation à l'antique n'est visible, à une exception près qui est d'ailleurs dans le corps de la missive et que l'expéditeur prend soin de traduire ${ }^{84}$.

S'il dote le plus souvent sa prose de citations et de références, soigneusement relevées par P. Santoni, celles-ci confirment l'impression d'avoir affaire à un recueil valorisant davantage la latinité sacrée que la latinité classique ${ }^{85}$. Sur un total d'environ 1270 citations ou références repérées, la part des auteurs de l'Antiquité païenne dépasse à peine $20 \%$. En tête l'Énéide suivi de Térence. En revanche, les grandes figures de la correspondance, Cicéron, Sénèque, Pline sont bien discrètes. Le reste relève de la culture biblique, vétérotestamentaire (près de $31 \%$ ), avec en tête les Psaumes, qui constituent près de la moitié des

${ }^{81}$ P. Santoni, «Les lettres de Nicolas de Clamanges à Gérard Machet », art. cité (n. 18), p. 795.

${ }^{82}$ D. Cecchetti, «Nicolas de Clamanges e Gérard Machet », art. cité (n. 2), p. 171, remarque que ce choix a été aussi opéré dans le petit recueil romain des lettres de Clamanges. Il s'oppose à la formule traditionnelle depuis les Pères jusqu'à Pétrarque, $a d$ plus accusatif.

${ }^{83}$ Exemple, lettre 309, BnF, ms. lat. 8577, fol. 75v : Lactee eloquentie et suavis facundie viro magistro M[artino] B[erruerii], decano Turonensi (« À maitre Martin Berruyer, doyen de Tours, homme plein du lait de l'éloquence et de suaves paroles »).

${ }^{84}$ Lettre 229, à l'évêque de Chartres, BnF, ms. lat. 8577, fol. $55 \mathrm{v}:$ A vestra reverenda paternitate nuper accepi pagellam unam ex Parisius $x^{a}$ sextilis, hoc est augusti («De vous, révérend père, j’ai reçu autrefois une lettre expédiée de Paris le $10^{\mathrm{e}}$ jour du sixième mois, c'est-à-dire août »). À noter que quand Jean de Montreuil fournit la date, ce qui est peu fréquent et toujours incomplet (pas de millésime), il le fait lui aussi de manière profane et vulgaire. Nicolas de Clamanges mixte les deux usages, si l'on en croit les sept lettres éditées par D. Cecchetti à la fin de son article.

${ }^{85}$ Grande est à cet égard la différence avec le recueil de Gaguin de cinquante ans plus tardif : ses références aux classiques sont deux fois plus nombreuses que ses références aux textes de la latinité sacrée. Mais aussi avec les références des lettres de Jean de Montreuil, qui donne à Cicéron, Virgile, Horace, Sénèque et Térence une place prépondérante, dépassant celle qu'occupent les références vétéroet néotestamentaires (voir les p. 377-401 de l'éd. Ornato). 
références tirées de l'Ancien Testament, puis loin derrière l'Ecclesiastique et Isaïe. La matière néotestamentaire représente un peu plus de $46 \%$, Matthieu étant nettement le plus cité des évangélistes. La patristique prend enfin une part singulièrement faible (à peine $2 \%$ ), quoique l'auteur ait été comparé à Augustin $^{86}$. Il est surtout intéressant de voir que près des deux tiers des références néotestamentaires proviennent des lettres de saint Paul, les Épîtres aux Corinthiens en premier lieu.

Cela donne sans doute la clef, à vrai dire assez déroutante, de la démarche du confesseur royal. Il ne s'agit pas de reprendre le modèle pétrarquien, même si certaines épîtres s'apparentent à des épîtres familières, mêlant les sujets, livrant des pensées intimes notamment sur la lassitude croissante de la vie de cour et sur une santé déclinante ${ }^{87}$, ce qui peut aussi les rattacher aux epistole seniles du Lauréat $^{88}$. Machet ne vise pas davantage la poursuite de l'œuvre épistolaire de Jean de Montreuil ou de Nicolas de Clamanges, même si sa démarche tire vraisemblablement son inspiration de ces grandes figures, de la dernière surtout, dont il rassembla sept lettres ${ }^{89}$. Mais si l'intention première du confesseur de Charles VII fut peut-être de les imiter, l'état final du recueil ne fait pas de celui-ci un « epistolario » humaniste. Il vise à témoigner non pas de la précellence des lettres françaises, mais plus modestement de l'implication du clerc dans ses devoirs d'évêque gallican, partisan de la réforme de l'Église. Aux 80 lettres écrites au diocèse de Castres, l'auteur affecte une sorte de pouvoir de représentation compensatoire de l'absentéisme du confesseur royal, absens corpore, presens spiritu ${ }^{90}$. Les lettres spirituelles adressées au chapitre castrais, les exhortations pieuses destinées à diverses personnes ou communautés, voire les conseils de patience donnés à quelques correspondants tombés en défaveur exhalent un parfum paulinien. Certes, Gérard Machet ne reprend pas les formes caractéristiques de l'épistolographie de l'Apôtre. Mais Paul imprègne en esprit tout son recueil.

\footnotetext{
${ }^{86}$ Lettre 32, BnF, ms. lat. 8577, fol. 13-13v.

${ }^{87}$ Lettre 390 et suiv., ibid., fol. 103 et suiv. santé.

${ }^{89}$ D. Cecchetti, « Nicolas de Clamanges e Gérard Machet », art. cité (n. 2), p. 144.

${ }^{90}$ Lettre 4, BnF, ms. lat. 8577, fol. 7.
}

${ }^{88}$ Pétrarque, Rerum senilium libri, Lettres de la vieillesse, Elvira Nota (éd.), Frédérique Castelli et al. (trad.), Paris, Les Belles Lettres, 5 vol., 2002-2013. Ce second «epistolario » du Lauréat couvre les quatorze dernières années de sa vie. L'amitié y tient une place importante, de même que les ennuis de 
Sans doute faut-il voir aussi, comme la matérialise la table du début, l'expression d'une fierté d'appartenance aux milieux navarristes. Pas moins de 100 lettres sont adressées à des membres passés ou présents du collège royal, dont l'évêque cultive le souvenir ou l'amitié. Et Gerson occupe une belle place parmi elles. Faire mémoire de tout cela importait prioritairement au prélat. L'amour des belles lettres, avec ou sans majuscule, n'est pas absent, Machet avait été à bonne école. Sans doute l'a-t-il mu dans un premier temps à constituer le recueil examiné. Mais là n'est pas la signification primordiale à donner à sa confection. Le produit «fini », supervisé par l'évêque de Castres, à défaut d'avoir été retravaillé à la manière d'un Nicolas de Clamanges, ne constitue assurément pas une œuvre littéraire illustrative du genre épistolaire.

Comme source, la correspondance de Gérard Machet fournit des données intéressantes à l'historien du monde ecclésiastique et de l'Église des années 1430-1440, décrits sous des dehors très sombres et assez convenus. Elle éclaire aussi les milieux universitaires au sortir de la grande crise des années 1413-1436. Elle donne à voir les réseaux d'entraide et leur fonctionnement. Elle fournit quelques éléments sur la personne et la politique de Charles VII. Elle pose surtout la question de son statut exact.

Il s'agit en réalité d'une correspondance assez peu épistolographique. En rien elle ne peut faire le pont ou la planche entre l'épistolographie du premier humanisme français, auquel Franco Simone rattachait Machet ${ }^{91}$, et celle du second. Encore sans doute imprégnée de l'ars dictaminis, visant peut-être à fournir à des manieurs de plume professionnels des formules modèles à utiliser dans telle ou telle circonstance, elle ne s'inscrit pas dans la «dialectique des rapports culturels franco-italiens ${ }^{92} »$. Dans l'esprit de son auteur, le recueil a pu être initialement conçu comme œuvre littéraire, mais sa fabrication peut-être mal maîtrisée et assurément non revue l'a bien éloigné de cet horizon. Le

\footnotetext{
${ }^{91}$ Franco Simone, Il Rinascimento francese, Studi e Ricerche, Turin, Societa Editrice Internazionale, 1961. Voir les critiques adressées à la vision trop étroite de cette période par Evencio Beltran, «L'humanisme français au temps de Charles VII et Louis XI », dans Carla Bozzolo et Ezio Ornato (dir.), Préludes à la Renaissance. Aspects de la vie intellectuelle en France au XV siècle, Paris, CNRS, 1992, p. 123-162, ici p. 126, 130.

${ }^{92}$ Gilbert Ouy, «La dialectique des rapports culturels franco-italiens et l'humanisme en France aux XIV et $\mathrm{XV}^{\mathrm{e}}$ siècles », dans Rapporti culturali ef economici fra Italia e Francia nei secoli dal XIV al XVI, Atti del colloquio italofrancese (Rome, 1978), Rome, Giunta centrale per gli studi storici, 1979, p. 137-157.
} 
manuscrit n'est peut-être qu'une ébauche que la mort du confesseur aurait empêchée de transformer en « epistolario » soigné.

Contrairement à celui de Clamanges, recopié et connu jusqu'au XVI siècle, il n'a pas fait autorité. Déposé dans la librairie de Saint-Martin de Tours, ville où Machet expira, il est tombé dans l'oubli. Robert Gaguin en ignore l'existence au moment de publier ses Epistole et orationes $^{93}$. Pourtant, quand le manuscrit entra dans les collections de Colbert, il fut rangé avec des volumes renfermant des recueils de lettres de Pétrarque, Cicéron, Sénèque, Pline, Jean de Salisbury, Pierre de la Vigne, Coluccio Salutati, Leonardo Bruni, Le Pogge et Pie $\mathrm{II}^{94}$. Mais c'est à tort que le Grand Siècle a flatteusement placé l'évêque de Castres en leur illustre compagnie.

Franck COLLARD Université Paris Nanterre

${ }^{93}$ Epistole et orationes Gaguini, Louis Thuasne (éd.), 2 t. en un vol., Paris, 1903, reprint Genève, Slatkine, 1977.

${ }^{94}$ Le Catalogue des manuscrits latins de la BnF, t. 4, cote ces manuscrits à partir de la cote lat. 8522 . 